\title{
Query Change as a Contextual Markov Model for Simulating User Search Behaviour
}

\author{
Saber Zerhoudi \\ University of Passau \\ Germany \\ saber.zerhoudi@uni-passau.de \\ Joerg Schloetterer \\ University of Duisburg-Essen \\ Germany \\ joerg.schloetterer@uni-due.de
}

\author{
Michael Granitzer \\ University of Passau \\ Germany \\ michael.granitzer@uni-passau.de \\ Christin Seifert \\ University of Duisburg-Essen \\ Germany \\ christin.seifert@uni-due.de
}

\begin{abstract}
Search engine users issue queries to formulate their information need and gain useful insights. However, it is challenging for search engines to understand different users' search type intents and return appropriate results. Simulating user search behaviour allows information retrieval systems (IR) to parameterise the a-priori distribution of search types using different back-end configurations and user interface variants to improve the retrieval functionality. In this paper, we propose a formal Markov approach in which we utilise the context discovery process to model user-type specific behaviour by capturing the user's query change in a search session. Contextual Markov models have been used in the past to improve the prediction of user intentions, we investigate here their efficiency in simulating user-type specific interactions. Additionally, we provide an empirical and classification-based evaluation that can be used in simulation assessment. Overall, we report that the proposed approach reliably simulates user-type specific behaviour on a real-world academic search engine log dataset.
\end{abstract}

\section{CCS CONCEPTS}

- Information systems $\rightarrow$ Personalization.

\section{KEYWORDS}

Simulating user interactions, query change, user search behaviour, simulation, information retrieval, Markov model

\section{ACM Reference Format:}

Saber Zerhoudi, Michael Granitzer, Joerg Schloetterer, and Christin Seifert. 2021. Query Change as a Contextual Markov Model for Simulating User Search Behaviour. In Forum for Information Retrieval Evaluation (FIRE 2021), December 13-17, 2021, Virtual Event, India. ACM, New York, NY, USA, 9 pages. https://doi.org/10.1145/3503162.3503165

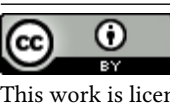

This work is licensed under a Creative Commons Attribution International 4.0 License.

FIRE 2021, December 13-17, 2021, Virtual Event, India

(C) 2021 Copyright held by the owner/author(s).

ACM ISBN 978-1-4503-9596-0/21/12.

https://doi.org/10.1145/3503162.3503165

\section{INTRODUCTION}

Analysing users' search behavior is key to understand how they interact with search engines and assess their experience. This analysis is essential to maintain a useful and usable information retrieval system (IR) that can modify its behaviour over time and for different users. It also provides guidance to the systems' design and evaluation such as deciding where each user interface component should be placed and which content should be provided to the user. Machine learning (ML) techniques have been applied to model user search behaviour by building global user interactions models [32] or models that group users into communities with common interests [17]. ML techniques are promising in cases where very large datasets are available. However, personalised log data are hard to obtain in search engines domain, and even when available, such models would be generated at a significantly smaller scale and would be less accurate. One other limitation is that this modelling approach describe only the correlations between data and lacks understandability (e.g., causal relationships between controlled inputs and corresponding outputs). To overcome such limitations, we propose to use simulation modelling.

Simulation offers a way to conduct a higher level of search and search behaviour analysis with explicit or non-existing factors (e.g., specific type of events or new design) which goes beyond the ML-modelling approach optimisation. Simulating user search behaviour also allows IR systems to conduct A/B-tests for the purpose of monitoring user interactions and parameterising the a-priori distribution of search types using different back-end configurations and user interface variants (e.g., changing facets placement in the user interface to more prominent place) to improve the retrieval functionality.

To better simulate users' search behaviour and model their information needs, search engines offer contextual information with data such as previous queries and click-actions, which is very useful for splitting users into subgroups sharing similar intentions. Therefore, in order to restrict our simulation modelling to the most promising contexts, we can either perform data exploration analysis or use domain expertise to choose an appropriate subgroup of contexts. However, acquiring a deep understanding of data by exploration or by domain expertise is often costly, especially for large-scale datasets. Thus, an alternative straightforward solution is to employ the commonly used search-type contexts (i.e., exploratory and lookup) and explore the work of Marchionini [21] and Kumaripaba 
et al. [1] which have introduced a set of indicators that characterise each search-type context to simulate personalised user search session. Although this solution has achieved promising performances in terms of predicting personalised user intents [18], it is still unclear whether it can simulate fine-grained user-type specific search sessions since users may express a mixed behaviour of exploratory and lookup while acquiring the knowledge needed to fulfil their information need. Therefore, we propose a method that utilises session-level query change as a contextual information.

Users frequently modify their search query in hope of retrieving better results. The previous-to-current query modification is called query reformulation or query change. Query change is an important form of feedback that monitors how user's information need evolves during a search session as they occur after the user examines search results. Thus, we derive a query change model that tracks the user's dynamic information need during a session to simulate contextaware personalised data.

Recently, there has been a growing interest in developing user search behaviour models and using simulation to address some of the limitations of ML techniques [16]. However, existing research only address the problem of simulating global search behaviour or superficial context-aware search behaviour (e.g., novices, occasional users and experts) [33]. Such an approach cannot capture well the contexts at its fine-granularity level (e.g., document ranking, query change..etc). One of the solutions is to simulate user search sessions using query change as contextual information.

In this work, we propose the use of Markov models to address the problem of simulating global and search-type specific behaviour to support users in their search. Our contributions are the following: (1) we conduct experiments on an academic real-world dataset and simulate global user search behaviour using first-order Markov approach which we consider as baseline, (2) we then simulate user-type specific behaviour using exploratory and lookup search contexts and we report the results and then (3) we showcase that simulating user-type specific behaviour using intent-level query change as context is highly effective at generating models with finer context granularity. We also provide (4) a detailed analysis of user's session-level query change behaviour on our dataset, (5) an empirical validation that utilises the Kolmogorov-Smirnov statistical test to compare the similarity between real log and simulated data distribution and (6) an evaluation technique that uses classification to assess the quality of simulated search session and calculate the context-aware models (i.e., exploratory-lookup and intent-level query change) improvement in comparison to the baseline.

\section{RELATED WORK}

Markov models are widely used for modelling user search behaviour as they are well-know for their simplicity, compactness and wellestablish theory. There are several existing approaches that apply Markov models to model user search behaviour: first-order Markov model [27], $k$ th-order Markov model, partially observable Markov decision process (POMDP) [30], hidden Markov models (HMM) [6] ..etc. While these approaches are effective in predictive modelling, we believe that first-order Markov models are well suited for simulation modelling without making it overly complex.
Several studies have shown that utilising context-awareness in session search modelling helps to better understand user information needs and improves the prediction accuracy of recommender systems [25] and query classification [4]. Early approaches for context-aware modelling focused on integrating the additional data, withdrawn from a given context, into the feature space and used it to learn hybrid or local models [31].

Our proposed work focuses on utilising query change as a contextual model. Query change has been heavily related to the problem of query suggestion [29] and query formulation [3, 9, 12]. Boldi et al. [3] derived a query flow graph, which is a graph that represents user's query behaviour, from user query logs to detect query chains and recommend queries based on different approaches (e.g. previous query, random walk, maximum weights..etc). Similarly, Guan et al. [8] used ontologies to study how queries move in the graphs. However, relying on large scale query logs may not be applicable to most of real-word scenarios due to the lack of query history data, and likewise, relying on ontology mapping is challenging [15] and tends to introduce inaccurate intermediate results and hurt the overall search accuracy.

Huang et al. [12] investigated different query change strategies (i.e. adding words, removing words, correcting the spelling, extending acronyms and substituting words) and reported that they more likely generate clicks, especially on higher-ranked results. However their approach does not address how to utilise query change types to improve information retrieval systems (IR). Liu et al. [20] explored the relationship between different task types and how user change their queries. They classified query change by intent-level types, namely, specification, generalisation, word repetition, word substitution and new topic. Yet, similarly to [12], authors did not expand their findings to improve IR systems.

Our discussion of related work reveals a strong focus on modelling user queries and their change. However, considering the user's query change process itself as a possible source of contextual information has often been neglected. In this work we focus on the evolution of user's querying pattern within sessions to study context-awareness using Markov models.

\section{APPROACH}

In this section, we first describe our baseline model to simulate global user search behaviour (Section 3.1), then we explore two different techniques as possible contexts to simulate user-type search behaviour: (1) classifying sessions according to their user navigation behaviour into two categories: exploratory and lookup (Section 3.2), and (2) grouping sessions by learning a mixture of intent-level query change characteristics (Section 3.3).

\subsection{User Search Behaviour Model}

Searching is a multi-step process where users interact with a search engine and seek to satisfy their information need. At each step, users may submit a new query. The information retrieval system (IR) retrieves the top- $k$ document sets that best match use's query. The top- $k$ documents are usually displayed in a result list with their title and a summary snippet. Users may click on one or more items in the list to retrieve the actual content and examine the document in detail. In case the user decides that the returned documents are 
insufficient, he/she may modify the query and examine the newly returned documents. This process continues until the user fulfils his/her information need.

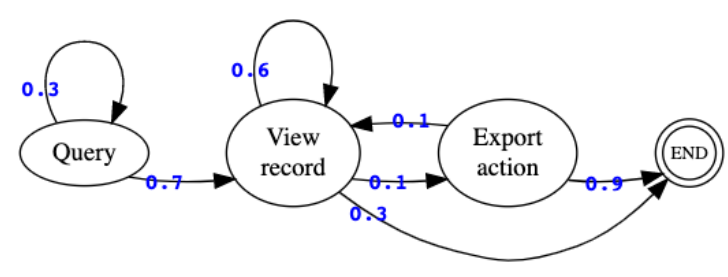

Figure 1: Example of a Markov model with multiple sequences of user search actions. Markov model is defined here as a user navigation graph with "Query" node referring to the query formulation, "View record" to when users click on a document, "Export action" to when users export a document and "END" marks the end of the sequence.

We propose investigating the use of Markov Chains to model the search dynamics. The theoretical model is based on first-order Markov models [27]. Our model is defined as a weighted directed graph, where user actions are represented as nodes and the transition probability between actions as edges as shown in Fig. 1. In fact, let $G=(V, E, w)$ where:

- $V$ is the set of all possible user search actions including the END node, with each action defined as state $A_{v}$,

- $E \subseteq V \times X$ is the set of all possible transitions between two actions,

- $w: E \rightarrow[0,1]$ is a function that assigns a weight $w$ to every pair of states $\left(A_{i}, A_{j}\right)$ which represents the likelihood of a transition from state $i$ to state $j$.

Let $X_{k}$ be the random variable that models actions in a user search session. The transition probability is modelled using maximum likelihood estimation:

$$
P\left(X_{k}=A_{j} \mid X_{k-1}=A_{i}\right)=\frac{N_{A_{i}, A_{j}}}{N_{A_{i}}}
$$

where $N_{A_{i}}$ is the total amount of how many times the action $A_{i}$ occurred in the training data and $N_{A_{i}, A_{j}}$ is the amount of how many times the transition from action $i$ to action $j$ has been observed.

\subsection{Contextual Markov Model}

During a search session, a user performs different search actions to find documents that fulfill his/her information need. The technique that we propose here aims to categorise users into different groups based on their search behaviour. Search tasks are commonly divided into two major types of user's behaviour [1,21]:

- Exploratory: where users are more likely to formulate more queries as they learn about the topic and explore the search result list exhaustively,

- Lookup: where users only investigate the first few results and rephrase their queries quickly.
Kumaripaba et al. [1] extended the work of Marchionini [21] and provided a few simple indicators of information search behaviours (e.g. query length, maximum scroll depth, completion time..etc) to categorise users into exploratory and lookup searchers. We utilise these indicators to split the training data into smaller portions. In fact, we build a first-order Markov model for each type (i.e. exploratory and lookup) and we compare them to the Markov model built from the whole data (i.e. Section 3.1). This would allow us to evaluate the impact of context on the accuracy of simulated sessions.

In this approach, the contextual model was developed with the exploratory-lookup distinction in mind, but it is well suited to accommodate other contexts as well [19]. In fact, given a training data $D$, we define $U=\left\{u_{1}, u_{2}\right\}$ as useful context that splits $D$ into $D_{1}$ and $D_{2}$ in a way that $M_{1}$ and $M_{2}$ are two Markov models learnt from $D_{1}$ and $D_{2}$ respectively.

\subsection{Query Change as Contextual Markov Model}

Users tend to generate search session with borderline behaviours and mixed characteristics. In fact, users may have precise search goals, yet the search process is not straightforward. Several studies have shown that the query change which occurs in a search session is heavily related to the contents of previously viewed search results $[13,28,34]$. Therefore, looking into how users change their query from $q_{i-1}$ to $q_{i}$ can partially explain how they express their dynamic information need in the session. As consequence, such terms may be used for categorising users into finer groups to better simulate search-type specific sessions.

In this approach, we expand the findings of Huang et al. [12] and adopt user's session-level query change process as an implicit indicator for categorising search behaviour and estimating the change in user's information need. We develop a syntactic taxonomy by analysing the query change $\Delta q_{i}$ as the syntactic change which occurs between two consecutive queries $q_{i-1}$ and $q_{i}$ :

$$
\Delta q_{i}=q_{i}-q_{i-1} \text {. }
$$

Let $W(q)$ be the bag of words for $q$ and $q_{i}, q_{i-1}$ two successive queries. A query $q_{i}$ can be decomposed into three main parts, namely theme, added and removed terms and written as follows:

$$
q_{i}=\Delta q_{i}^{=}+\Delta q_{i}^{+}-\Delta q_{i}^{-}
$$

where:

$$
\begin{aligned}
& \Delta q_{i}^{=}=\left\{w \mid w \in W\left(q_{i}\right), w \in W\left(q_{i-1}\right)\right\} ; \\
& \Delta q_{i}^{+}=\left\{w \mid w \in W\left(q_{i}\right), w \notin W\left(q_{i-1}\right)\right\} ; \\
& \Delta q_{i}^{-}=\left\{w \mid w \notin W\left(q_{i}\right), w \in W\left(q_{i-1}\right)\right\} .
\end{aligned}
$$

We refer to the common terms that appear in both query $q_{i}$ and $q_{i-1}$ as theme terms and denote it $\left(\Delta q_{i}^{=}\right)$as they usually represent the main thematic of a session. Added terms $\left(\Delta q_{i}^{+}\right)$represent the new terms that users add to the previous query and removed terms $\left(\Delta q_{i}^{-}\right)$refer to the terms that users delete from the previous query as they seek to improve bad performing queries.

Theme terms $\left(\Delta q_{i}^{=}\right)$are generated using the longest common subsequence (LCS) approach [11] in both queries $q_{i}, q_{i-1}$. The LCS can be the common part or parts of two queries as long as the subsequence appears in both queries in the same relative order but not necessarily continuous. For instance, $S_{1}: q_{1} \longrightarrow q_{2}$ is a search 
session where $q_{1}=$ "tire recycling technique in Europe" and $q_{2}=$ "tire recycling facilities in Europe". $\Delta q_{i}^{=}=$"tire recycling in Europe", $\Delta q_{i}^{+}=$"facilities" and $\Delta q_{i}^{-}=$"technique".

We design a granular categorisation of five major query change classes based on the previous terms:

- Addition: $\Delta q_{i}^{+} \neq \emptyset, \Delta q_{i}^{-}=\emptyset$;

- Removal: $\Delta q_{i}^{+}=\emptyset, \Delta q_{i}^{-} \neq \emptyset$;

- Change: $\Delta q_{i}^{+} \neq \emptyset, \Delta q_{i}^{-} \neq \emptyset, \Delta q_{i}^{=} \neq \emptyset$;

- Repetition: $\Delta q_{i}^{+}=\emptyset, \Delta q_{i}^{-}=\emptyset, \Delta q_{i}^{=} \neq \emptyset$;

- Others: $\Delta q_{i}^{+} \neq \emptyset, \Delta q_{i}^{-} \neq \emptyset, \Delta q_{i}^{=}=\emptyset$;

Since the aim here is to capture major query intent change by looking only at syntactic query change between two consecutive queries, we deliberately exclude minor query change types such as "spelling correction" which may not be considered as a word-level syntactic change.

In this approach, the contextual model is developed using the above-mentioned major query change classes.

\section{EXPERIMENTAL SETUP}

The focus of our experiments is to determine whether contextaware methods improves the simulation of user-type specific behaviour. We first briefly introduce our dataset (Section 4.1). We then describe our models (Section 4.2) and finally present our evaluation setting (Section 4.3).

\subsection{Dataset}

In order to examine users' query change behaviors, we use Sowiport ${ }^{1}$ User Search Session Data Set (SUSS) ${ }^{2}$ [22] for our experiments, which includes 484,437 individual search sessions, 179,796 queries and around 8 million log entries that was collected over a period of one year (from April 2014 to April 2015).

Sowiport describes users' search actions using a list of 58 different actions that covers all user's activities while interacting with the interface of the search engine (e.g., formulating a query, clicking on a document, viewing the full document's content, selecting a facet, using search filters, etc.). For each user interaction, a session id, date stamp, length of the action and other additional information are stored to describe user's path during the search process. The dataset uses a threshold of 20 minutes of inactivity to determine the end of a session.

Table 1: Example of a search session for a specific user.

\begin{tabular}{ccc} 
Date stamp & Action label & Action length \\
\hline 2014-09-28 10:45:18 & goto_home & 19 \\
2014-09-28 10:45:37 & goto_advanced_search & 36 \\
2014-09-28 10:46:13 & query_form & 86 \\
2014-09-28 10:47:39 & search_advanced & 58 \\
2014-09-28 10:48:37 & view_record & 40 \\
2014-09-28 10:49:17 & view_record & 29 \\
2014-09-28 10:49:46 & search_change_paging & 28 \\
2014-09-28 10:50:14 & view_record & 26 \\
2014-09-28 10:50:40 & view_description & 106
\end{tabular}

\footnotetext{
${ }^{1}$ http://www.sowiport.de

${ }^{2}$ The dataset is publicly available at http://dx.doi.org/10.7802/1380
}

In Table 1, we showcase an example of a recorded user search session. In this session, the user started the session by visiting the homepage "goto_home" and the advanced search page, he/she then submitted a query "query_form" and performed an advanced search task "search_advanced". After loading the search result page, the user goes through the results and decides to view two documents "view_record". Unsatisfied with the results presented in the first page, he/she decides then to look for other results in the next search result page "search_change_paging" where he/she view a document "view_record" and read its abstract "view_description". The action length refers to the time spent by a user in each action.

From the 484,437 individual search sessions in the dataset, we remove sessions that do not contain a query (i.e., users having searched nothing) or have invalid query annotations and we randomly sample 100,000 sessions which we refer to as SUSS $^{-}$.

\subsection{Models}

We here detail the models used in our evaluation.

4.2.1 Baseline. We use first-order Markov model as a baseline to simulate user search sessions where each user action corresponds to a state in the model as shown in Fig. 1. We compute the transition probabilities using the SUSS $^{-}$search sessions.

4.2.2 Contextual Markov Model. We aim to use contextual information as a way to improve the simulation ability of Markov models. Therefore, we build a first-order Markov model for each context and compare it to the Markov model built for the whole data (Subsection 4.2.1). In this approach, we utilise the commonly used search-type contexts (i.e., exploratory and lookup) as contextual information.

Athukorala et al. [1] showed that the most distinctive indicators that characterise exploratory search behaviours are query length, maximum scroll depth, and task completion time. They proved empirically that exploratory tasks can be distinguished within in the first query session by these indicators. Following their findings, we identify lookup search sessions by capping the first query duration to 200 seconds, the dwell duration of the first three actions to 120 seconds, the cumulative actions tally in the first query iteration to 4 actions and the the task completion time to 800 seconds. The remaining user sessions are considered as exploratory search.

As a result of applying the exploratory-lookup-based approach to the SUSS $^{-}$dataset, we notate $39.42 \%$ sessions as lookup search type and the remaining as exploratory.

4.2.3 Query Change as Contextual Markov Model. Similarly to the previous approach, we utilise contextual information to improve the simulation ability of Markov models using the same process. We use the query change classes defined in Section 3.3 as contextual information. In particular, following Huang et al. [12] findings, we define five intent-level query change types:

- Specification: Adding one or multiple keywords into the original query $q_{i-1} \subseteq q_{i}$, i.e. "addition" query change class;

- Generalisation: Removing one or multiple keywords from the original query $q_{i} \subseteq q_{i-1}$, i.e. "removal" query change class;

- Word Substitution: Removing or adding one or multiple keywords, i.e. "change" query change class; 
- Word Repetition: Issuing similar queries $q_{i}=q_{i-1}$, i.e. "repetition" query change class;

- New Topic: Issuing very different queries $q_{i} \cup q_{i-1}=\emptyset$, i.e. "others" query change class.

These intent-level query change types will serve as searchtype contexts. For simplicity reasons, we assume that a user is always associated with one contextual class and contexts are nonoverlapping. We experiment labelling sessions with the intent-level class recorded in the first query change and with the most common intent-level class recorded in the entire session and compare the results.

In this approach, only sessions that contain at least two queries are considered (i.e., $87.37 \%$ of the SUSS$^{-}$dataset).

\subsection{Experimental design}

The quality of simulated user search sessions is usually evaluated by comparing real log and simulated data. In fact, simulated data are expected to be similar to real data as we do not want them to be distinguishable. In this section, we first evaluate our approach through a Kolmogorov-Smirnov test to determine if the real and simulated data belong to the same distribution (Subsection 4.3.1). Then we use a classification test to evaluate the simulation performance of our context-aware models and calculate their improvement in comparison to the baseline (Subsection 4.3.2).

4.3.1 Kolmogorov-Smirnov-based Evaluation. The two-sample Kolmogorov-Smirnov (KS-2) goodness-of-fit test [14] is one of the most useful and non-parametric methods for comparing two datasets. It is a convenient method for investigating whether two probability distributions can be regarded as indistinguishable.

In this paper, we utilise the two-sample KS to compare simulated data distribution to real log data for each model in Section 4.2. We derive seven separate simulation models for context-aware approaches (i.e., exploratory, lookup, specification, generalisation, word substitution, word repetition, new topic) and one global simulation model that is trained on whole dataset.

For example, in the exploratory-lookup-based approach, we divided the SUSS $^{-}$dataset into lookup and exploratory subsets (i.e., lookup real log data and exploratory real log data) and for each subset, we constructed a Markov model to simulate user-type specific search sessions (i.e., lookup simulated data and exploratory simulated data). In order to investigate, for instance, whether lookup real log data and lookup simulated data belong to the same distribution, we use the transition probabilities between states, each as a data point, drawn from the log sessions and the simulated sessions separately to create two independent samples.

Essentially, we test the null hypothesis that the two independent samples are drawn from the same distribution and proceed with calculating the absolute value of the distance between two data samples which we refer to as the test statistic $d$ to compare their distribution for similarities. The test statistic $d$ is calculated as follow:

$$
d=\sup _{x}\left|E_{n 1}(x)-E_{n 2}(x)\right|
$$

where, $n 1$ and $n 2$ are observations from the first and second sample respectively. $E_{n 1}(x)$ and $E_{n 2}(x)$ are the empirical distributions of the first and second sample, obtained from $\mathrm{n} 1$ and $\mathrm{n} 2$ observations.
We then compare the test statistic value against the critical value derived from the KS-2 table value to either accept or reject the hypothesis. The null hypothesis is accepted when the test statistic value (eq.1) is less than the table value and rejected otherwise.

Likewise, the critical value for the two samples can be approximated using the following formula:

$$
D_{n 1, n 2}=c(\alpha) \sqrt{\frac{n 1+n 2}{n 1 \cdot n 2}}
$$

where $\alpha$ refers to the level of significance $(\alpha=0.01)$ and $n 1, n 2$ are observations from the first and second sample respectively.

4.3.2 Classification-based Evaluation. In addition to the KS-2 test, we define a classification-based evaluation to (1) evaluate the simulation performance of our context-aware models and (2) calculate their improvement in comparison to the baseline.

In order to evaluate the simulation performance of our contextaware models, we first develop a set of features that represent the sequentiality of a user search session in the form of a feature vector. Then we train a classifier to distinguish simulated sessions from real $\log$ data sessions and report the results.

Inspired by the findings in [10] about what kinds of engineered features are best suited to various machine learning model types, we developed a set of features that represent the sequentiality of the search session (i.e., query search types; search types; advanced search types; clicking, viewing and exporting actions..etc) and discarded those that only describe the user's overall search behaviour (e.g., tally of search actions, queries formulation and clicks).

We used a binary vector to indicate the presence of a feature (i.e., (0) if present and (1) if not) and ordered features in the sequence (i.e., $i$ ffeature where $i$ refer to the sequence order of the query in a session, e.g., 1_search, 2_view_record). The resulting feature vector has a higher dimensionality (170) than the raw feature categories (58) due to the inclusion of the sequence order in the binary feature vector. Fig. 2 gives an example of a user session in the form of a feature vector.

$$
\begin{aligned}
& \text { [ "1_search", "1_search_change_paging", "1_view_record", } \\
& \text { "2_search", "2_search_change_paging", "3_search", } \\
& \text { "3_view_record", "3_view_description", "end"] }
\end{aligned}
$$

Figure 2: Example of a user search session in the form of a feature vector.

Each user session is converted to a feature vector, labelled and fed to a classifier. This process was repeated separately for each of the Markov approaches, namely, baseline, contextual and its query change variant. We created an equal amount of simulated sessions as real log sessions for a balanced classification and evaluated five classifiers with 10-fold cross-validation. As per the classifier, we used the five most popular algorithms in binary classification, namely, Logistic Regression [26], Support Vector Machine [23], K-Nearest Neighbors [5], Decision Trees [24], Random Forest [2] and we reported the average score. We also used automated machine learning (Auto-sklearn [7]) as it employs an ensemble of top performing models discovered during the optimisation process.

In order to calculate improvements of context-aware models in comparison to the baseline, we utilise metrics such as Precision 
(the proportion of the predicted correct labels over the number of predicted labels), Recall (the proportion of the predicted correct labels over the number of actual labels), F-measure (the harmonic mean of precision and recall) and Accuracy (the proportion of predicted correct labels over the total number of predicted and actual labels) which are common for objectively measuring the classifier's performance. In our case, we consider True Positive (TP) to be the scenario where the model classifies simulated sessions as simulated. A score of 0 means that the classifier cannot distinguish between simulated and real log sessions and therefore the simulated sessions are similar to real $\log$ data sessions, whereas with a score of 1 , simulated sessions and log data are completely different.

Since we can distinguish between real log and simulated sessions, reporting the accuracy alone can obfuscate some of the performance that F-measure would highlight. F-measure tells how precise the classifier is (i.e., how many instances it classifies correctly), as well as how robust it is (i.e., does not miss a significant number of instances). In fact, if F-measure showed low precision/recall along with a low accuracy, we can have better confidence in the results. Therefore, we utilise all four metrics to demonstrate relative performance and consistency of the results.

\section{RESULTS}

It is important to investigate users' session-level query change to find heuristics for providing insights and designing better evaluation metrics. Therefore, we first provide a detailed analysis of session-level query change behavior and then discuss our experimental results.

\subsection{Query Change Analysis}

A search session consist of a sequence of queries where the query count may change depending on users' intent shift. In order to explore the performance across sessions with different lengths, we divide all our sessions into three different groups according to the number of queries: (1) short sessions (with two queries), (2) medium sessions (with three to four queries) and (3) long sessions (with at least five queries). The SUSS ${ }^{-}$dataset contains $16.03 \%$ of short sessions, $36.27 \%$ of medium sessions and $47.69 \%$ of long sessions, which indicates that in the Sowiport users tend to submit multiple queries to fulfil their information need. The distribution of queries in search sessions is shown in Fig.3.

Next, we analyse the distribution of search sessions after labelling them using (1) the intent-level class recorded in the first query change and (2) the most common intent-level class recorded in the entire session. According to Table 2, the most frequent intentlevel query change types are "New Topic", "Word Substitution", "Word Repetition" and "Specification". This can indicates that users tend to narrow down their search scope by adding restrictions or specifications to the initial query or tend to gradually shift their intents to other subtopics by replacing some terms from the previous queries or by issuing different queries. On the other hand, we find that "Generalisation" intent-level types is the least frequent mainly due to the fact that users rarely decide to scale up their search scope by removing words from the initial query.

In addition, table 2 shows that we can rely on the first query change to determine the overall intent-level class for each sessions.

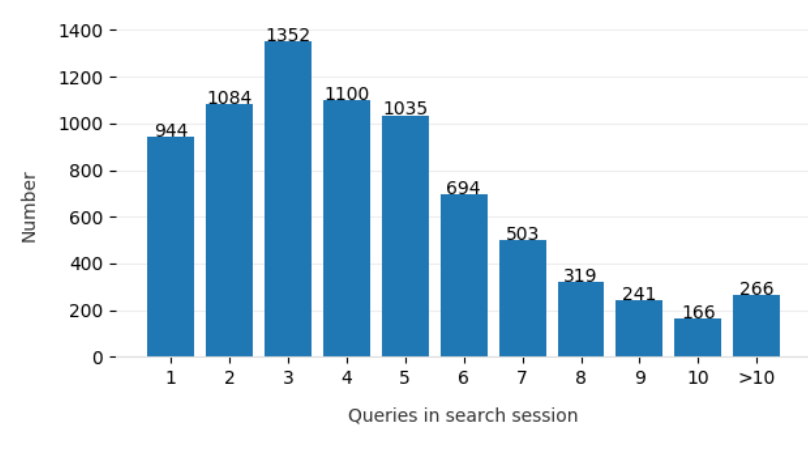

Figure 3: Distribution of queries in search sessions (session length) in SUSS $^{-}$dataset.

Table 2: Distribution of search session after labelling them using (1) the intent-level class recorded in the first query change and (2) the most common intent-level class recorded in the entire session.

\begin{tabular}{lcc}
\hline Approach & \multicolumn{2}{c}{$\begin{array}{c}\text { Query change } \\
\text { First }\end{array}$} \\
& 1 & 1 \\
\hline Most common \\
\hline Specification & 0.164 & 0.138 \\
Generalisation & 0.081 & 0.157 \\
Word Substitution & 0.209 & 0.191 \\
Word Repetition & 0.189 & 0.175 \\
New Topic & 0.354 & 0.339 \\
\hline
\end{tabular}

Therefore, we use the distribution we obtained from the first query change for the remaining experiments.

\subsection{User Search Behaviour Simulation}

5.2.1 Kolmogorov-Smirnov-based Evaluation. For this evaluation test, we derived seven separate simulation models (i.e., two from exploratory-lookup approach and five from the intent-level query change approach) and one global simulation model that is trained on whole SUSS ${ }^{-}$dataset. For each model, we utilise the transition probabilities between states which are drawn from the log sessions and the simulated sessions separately to generate two independent samples. By feeding these data points to the given formula (1) we obtain the test statistic value and compare it to the critical value (2) for the two samples. We also report the p-value which provides a measure of how much we can trust the statistical test. In general, the closest the p-value to zero is, the more reliable the test is.

Table 3 shows that the statistical value is smaller than the critical value across all models, hence we retain the null hypothesis. Therefore, we conclude that the simulated and the real log sessions belong to the same distribution.

5.2.2 Classification-based Evaluation. Since the KS-2 critical values are all significant, it means that query change as context factor does not improve the simulation or at least it is hard to quantify the improvement using a KS-2 test. Therefore, we need to adopt a second evaluation method: we investigate whether we can train a 
Table 3: Two-sample Kolmogorov-Smirnov Test of $\log$ and simulated sessions distributions using baseline and Contextual Markov model (CMM) approaches. The p-value provides a measure of how much we can trust the statistical test (the closest the p-value to zero is, the more reliable the test is).

\begin{tabular}{llcc}
\hline \multicolumn{1}{c}{ Approach } & \multicolumn{2}{c}{ Kolmogorov-Smirnov Test } \\
& & D-statistic $(\mathbf{p}$-value $)$ & D-critical \\
\hline Baseline & $0.00417(7.44 e-11)$ & 0.00421 \\
\hline CMM & Exploratory & $0.00381(6.54 e-12)$ & 0.00389 \\
& Lookup & $0.00302(4.11 e-12)$ & 0.00356 \\
\hline \multirow{4}{*}{ CMM } & Specification & $0.00298(2.56 e-12)$ & 0.00314 \\
& Generalisation & $0.00387(3.88 e-12)$ & 0.00391 \\
& Substitution & $0.00335(3.72 e-12)$ & 0.00341 \\
& Repetition & $0.00358(3.16 e-12)$ & 0.00364 \\
& New Topic & $0.00326(3.57 e-12)$ & 0.00333 \\
\hline
\end{tabular}

classifier and try to distinguish between real log and simulated sessions through controlled scenarios. For each scenario, we simulate an equal amount of sessions as present in the log data to balance class distribution.

Table ?? shows that when using contextual Markov with the exploratory-lookup approach, the model did better while simulating sessions for "Lookup" with an F-measure score of 0.509 in comparison to "Exploratory" with a score of 0.568 . One possible explanation for this is that lookup sessions are probably easier to simulate since there is less variation. The exploratory group of users generate longer sessions, thus higher total of state transitions which results a diverse number of simulated sessions. In addition, table ?? also reports low precision (i.e., 0.573 for "Lookup" and 0.636 for "Exploratory")/recall (i.e., 0.487 for "Lookup" and 0.527 for "Exploratory") values along with a low accuracy score (i.e., 0.564 for "Lookup" and 0.608 for "Exploratory") when using the exploratorylookup approach in comparison to the baseline, which indicates that we can have better confidence in the results.

On the other hand, table ?? illustrates that when using contextual Markov with the query-change approach, the model produced fairly low F-measure results while simulating sessions for "Word Repetition" (0.490), "New Topic" (0.475), "Word Substitution" (0.502) and "Specification" (0.453) in comparison to "Generalisation" (0.562). In parallel, table ?? also reports low precision/recall values along with a low accuracy score across all the context-aware models which indicates that we can have better confidence in the results.

Overall, table 4 shows that both contextual Markov models (CMM), exploratory-lookup and query change, achieve higher performance in comparison to the baseline. In particular, the models performed better on an overall level with a weighted average of F-measure score of 0.532 for exploratory-lookup and 0.485 for query change and the relative improvement of the exploratory-lookup model scored $29.07 \%$ in comparison to the baseline, while the query change model achieved a score of $35.25 \%$. The relative improvement (RI) represents the relative change between the baseline and the weighted average of all the context-aware models (W.Sum).

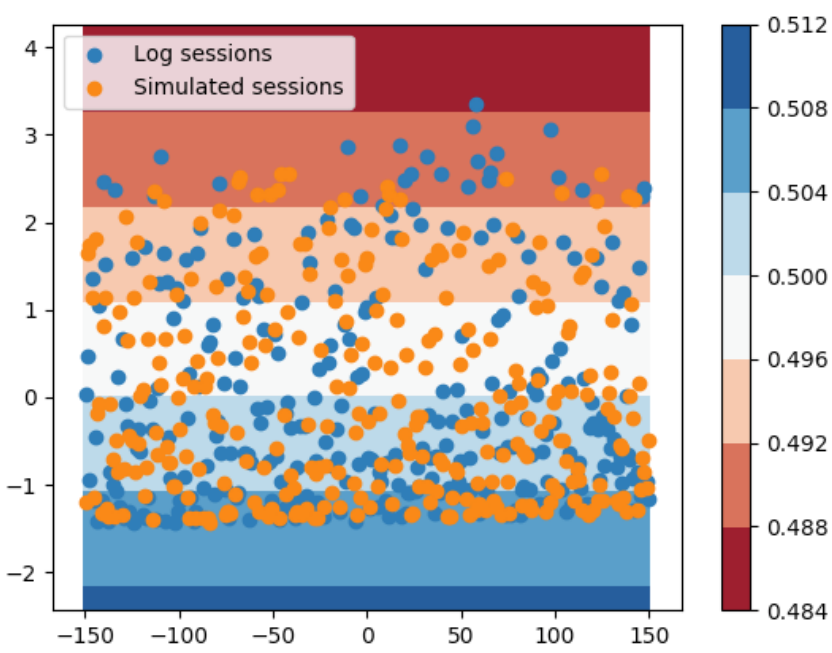

Figure 4: Probability Decision Surface for Logistic Regression on our binary classification using PCA. Simulated sessions are generated using contextual Markov model (lookup search type).

This means that the accuracy of the classifier has been reduced by $29.07 \%$ while using the exploratory-lookup approach and by $35.25 \%$ while using the intent-level query change approach. We also remind that reducing the accuracy of the classifier means it find it hard to distinguish between simulated and real log sessions and therefore the simulated sessions are similar to real $\log$ data sessions.

In summary, we report that grouping user search sessions depending on their behavioural characteristics helps improving the simulation quality. In fact, using intent-level query change contextual information helped creating simulated sessions more similar to original ones (i.e., reducing the accuracy of the classifier which is translated by lower F-measure, recall and precision values).

To gain further insights, we visually analysed the decisions made by the Logistic Regression classifier by projecting them onto a 2dimensional space (cf. Fig. 4). The idea here is to monitor how the classifier decides to divide the feature space by class label (i.e., log data sessions and simulated sessions using contextual Markov model). We used Principal Component Analysis (PCA) to generate two compound features which are created to capture as much of the variance in our original 170 features as possible. We noticed that the classifier is unsure (brighter colors) around the middle of the domain and very confident (darker colors) in the bottom and top parts of the domain. We also note that the sampling points in the feature space are mainly spread over the middle, where the classifier is the most unsure. Furthermore, the classifier did not succeed in setting up a clear separation between samples from the two classes. 
Table 4: Classification of real $\log$ sessions vs simulated sessions using baseline and contextual Markov model (CMM) approaches. We report the accuracy, recall, precision and F-measure across $10-\mathrm{CV}$ folds (while (1) averaging over five classifiers defined in subsection 4.3.2 and (2) using Auto-sklearn. W.Sum is the weighted average score and RI is the relative improvement compared to the baseline. Bold indicates the best result in terms of the corresponding metric. Lowest results are the best as we aim to reduce the classifier's capability to distinguish between real log and simulated sessions.

\begin{tabular}{l|c|cc|cc|cc|cc}
\hline \multicolumn{1}{c}{ Approach } & \multirow{2}{*}{ Size } & \multicolumn{2}{c}{ Accuracy } & \multicolumn{2}{c}{ Recall } & \multicolumn{2}{c}{ Precision } & \multicolumn{2}{c}{ F-measure } \\
& & Avg. & AutoSklearn & Avg. & AutoSklearn & Avg. & AutoSklearn & Avg. & AutoSklearn \\
\hline Baseline & 1 & 0.661 & 0.660 & 0.773 & 0.764 & 0.756 & 0.748 & 0.750 \\
\hline CMM Exploratory & 0.39 & 0.608 & 0.612 & 0.527 & 0.531 & 0.636 & 0.631 & 0.568 & 0.746 \\
& 0.61 & $\mathbf{0 . 5 6 4}$ & $\mathbf{0 . 5 6 8}$ & $\mathbf{0 . 4 8 7}$ & $\mathbf{0 . 4 8 6}$ & $\mathbf{0 . 5 7 3}$ & $\mathbf{0 . 5 7 7}$ & $\mathbf{0 . 5 0 9}$ & $\mathbf{0 . 5 2 8}$ \\
\hline W.Sum & 1 & 0.581 & 0.585 & 0.502 & 0.503 & 0.597 & 0.598 & 0.532 & 0.546 \\
\hline RI & - & $\mathbf{1 2 . 0 8 \%}$ & $\mathbf{1 1 . 3 4 \%}$ & $\mathbf{3 4 . 9 8 \%}$ & $\mathbf{3 4 . 0 9 \%}$ & $\mathbf{2 0 . 9 6 \%}$ & $\mathbf{2 0 . 0 5 \%}$ & $\mathbf{2 9 . 0 7 \%}$ & $\mathbf{2 6 . 7 1 \%}$ \\
\hline
\end{tabular}

(a) Exploratory-lookup context-aware models

\begin{tabular}{|c|c|c|c|c|c|c|c|c|c|c|}
\hline \multirow{2}{*}{\multicolumn{2}{|c|}{ Approach }} & \multirow[t]{2}{*}{ Size } & \multicolumn{2}{|c|}{ Accuracy } & \multicolumn{2}{|c|}{ Recall } & \multicolumn{2}{|c|}{ Precision } & \multicolumn{2}{|c|}{ F-measure } \\
\hline & & & Avg. & AutoSklearn & Avg. & AutoSklearn & Avg. & AutoSklearn & Avg. & AutoSklearn \\
\hline Baselir & & 1 & 0.661 & 0.660 & 0.773 & 0.764 & 0.756 & 0.748 & 0.750 & 0.746 \\
\hline \multirow{5}{*}{ CMM } & Specification & 0.164 & 0.453 & 0.450 & 0.455 & 0.425 & 0.451 & 0.467 & 0.453 & 0.445 \\
\hline & Generalisation & 0.081 & 0.551 & 0.536 & 0.528 & 0.589 & 0.601 & 0.591 & 0.562 & 0.590 \\
\hline & Substitution & 0.209 & 0.486 & 0.495 & 0.528 & 0.523 & 0.479 & 0.472 & 0.502 & 0.496 \\
\hline & Repetition & 0.189 & 0.485 & 0.481 & 0.504 & 0.501 & 0.476 & 0.483 & 0.490 & 0.492 \\
\hline & New Topic & 0.354 & 0.460 & 0.454 & 0.483 & 0.496 & 0.468 & 0.477 & 0.475 & 0.486 \\
\hline \multicolumn{2}{|c|}{ W.Sum } & 1 & 0.475 & 0.472 & 0.493 & 0.496 & 0.478 & 0.483 & 0.485 & 0.489 \\
\hline \multicolumn{2}{|l|}{ RI } & - & $28.14 \%$ & $28.44 \%$ & $36.10 \%$ & $34.95 \%$ & $36.72 \%$ & $35.39 \%$ & $35.25 \%$ & $34.37 \%$ \\
\hline
\end{tabular}

(b) Intent-level query change context-aware models

\section{DISCUSSION AND FUTURE WORK}

Although these results do not undoubtfully prove that we succeeded in simulating look-alike user search sessions, they show that users in our simulated sessions behave in a way that comes closer to the original behaviour from the real log data.

A main contribution of our approach is that we study changes among query transitions and utilise it to model the dynamics of search sessions. In a search session, users tend to modify their query and assess different results in order to fulfill their information need leaving a recurring search footprint query $\longrightarrow$ clicks $\longrightarrow$ query $\longrightarrow \ldots$ in the logs.

As different users may have different backgrounds and habits while using search engines, more information regarding how users' search intent evolve during a session can be considered to better simulate specific user search sessions. Therefore, as an extension to this study, we aim to extend our approach - where we simplified session labels to a fixed search intent - to cover evolving search intent where users can be associated to more than one intent-level query change class during a single search session Specification $\longrightarrow$ Word Substitution $\longrightarrow$ New Topic.

\section{CONCLUSION}

In this paper, we used context-aware methods to simulate usertype specific behaviour. We provided evidence that if we can apply exploratory analysis to identify useful contextual features, the contextual models are expected to reliably simulate specific search behaviour. In particular, we explored two different techniques as possible contexts for the domain: (1) classifying users according to their navigation behaviour into two categories: exploratory and lookup, and (2) grouping users by learning a mixture of intent-level query change characteristics into five categories (i.e., specification, generalisation, word substitution, word repetition and new topics).

We performed experiments using a real-world academic dataset with contextual Markov models and provided empirical results showing that it outperforms the first-order baseline in simulating similar user search sessions. The empirical results show that the context-aware models using query change as context type allow to account for finer context granularity, i.e., more specific models. In particular, utilising query change as contextual information allows us to better simulate sessions that demonstrate evolution and exploration of user's dynamic information need in the session.

\section{ACKNOWLEDGMENTS}

This work has been partially carried out within the project "SINIR: Simulating INteractive Information Retrieval" funded by the DFG (grant HA 5851/3-1).

\section{REFERENCES}

[1] Kumaripaba Athukorala, Dorota Głowacka, Giulio Jacucci, Antti Oulasvirta, and Jilles Vreeken. 2016. Is Exploratory Search Different? A Comparison of Information Search Behavior for Exploratory and Lookup Tasks. 7. Assoc. Inf. Sci. Technol. 67, 11 (Nov. 2016), 2635-2651. https://doi.org/10.1002/asi.23617 
[2] Mariana Belgiu and Lucian Drăgut. 2016. Random forest in remote sensing: A review of applications and future directions. ISPRS fournal of Photogrammetry and Remote Sensing 114 (04 2016), 24-31. https://doi.org/10.1016/j.isprsjprs.2016. 01.011

[3] Paolo Boldi, Francesco Bonchi, Carlos Castillo, Debora Donato, Aristides Gionis, and Sebastiano Vigna. 2008. The query-flow graph: model and applications. In Proceedings of the 17th ACM conference on Information and knowledge management. 609-618.

[4] Huanhuan Cao, Derek Hao Hu, Dou Shen, Daxin Jiang, Jian-Tao Sun, Enhong Chen, and Qiang Yang. 2009. Context-aware query classification. In Proceedings of the 32nd international ACM SIGIR conference on Research and development in information retrieval. $3-10$

[5] Sahibsingh A. Dudani. 1976. The Distance-Weighted k-Nearest-Neighbor Rule IEEE Transactions on Systems, Man, and Cybernetics SMC-6, 4 (1976), 325-327. https://doi.org/10.1109/TSMC.1976.5408784

[6] Sean R Eddy. 1996. Hidden markov models. Current opinion in structural biology 6, 3 (1996), 361-365.

[7] Matthias Feurer, Aaron Klein, Katharina Eggensperger, Jost Tobias Springenberg, Manuel Blum, and Frank Hutter. 2019. Auto-sklearn: Efficient and Robust Automated Machine Learning. Springer International Publishing, Cham, 113-134. https://doi.org/10.1007/978-3-030-05318-5_6

[8] Dongyi Guan and Hui Yang. 2013. Increasing stability of result organization for session search. In European Conference on Information Retrieval. Springer, 471-482.

[9] Fan Guo, Chao Liu, Anitha Kannan, Tom Minka, Michael Taylor, Yi-Min Wang, and Christos Faloutsos. 2009. Click chain model in web search. In Proceedings of the 18th international conference on World wide web. 11-20.

[10] Jeff Heaton. 2016. An empirical analysis of feature engineering for predictive modeling. SoutheastCon 2016 (Mar 2016). https://doi.org/10.1109/secon.2016. 7506650

[11] Daniel S. Hirschberg. 1977. Algorithms for the Longest Common Subsequence Problem. F. ACM 24, 4 (Oct. 1977), 664-675. https://doi.org/10.1145/322033. 322044

[12] Jeff Huang and Efthimis N Efthimiadis. 2009. Analyzing and evaluating query reformulation strategies in web search logs. In Proceedings of the 18th ACM conference on Information and knowledge management. 77-86.

[13] Jiepu Jiang and Chaoqun Ni. 2016. What Affects Word Changes in Query Reformulation During a Task-based Search Session? 111-120. https://doi.org/10.1145/ 2854946.2854978

[14] Frank J. Massey Jr. 1951. The Kolmogorov-Smirnov Test for Goodness of Fit. Amer. Statist. Assoc. 46, 253 (1951), 68-78. https://doi.org/10.1080/01621459.1951. 10500769

[15] Yannis Kalfoglou and Marco Schorlemmer. 2003. Ontology mapping: the state of the art. The knowledge engineering review 18, 1 (2003), 1-31.

[16] Heikki Keskustalo and Kalervo Järvelin. 2010. Simulations as a means to address some limitations of laboratory-based ir evaluation. The fanus Faced Scholar: A Festschrift in Honour of Peter Ingwersen (2010).

[17] Julia Kiseleva. 2015. Using Contextual Information to Understand Searching and Browsing Behavior. In Proceedings of the 38th International ACM SIGIR Conference on Research and Development in Information Retrieval (Santiago, Chile) (SIGIR '15). Association for Computing Machinery, New York, NY, USA, 1059.

[18] Julia Kiseleva, Hoang Thanh Lam, Mykola Pechenizkiy, and Toon Calders. 2013 Predicting Current User Intent with Contextual Markov Models. In 2013 IEEE 13th International Conference on Data Mining Workshops. 391-398.

[19] Julia Kiseleva, Hoang Thanh Lam, Mykola Pechenizkiy, and Toon Calders. 2013. Predicting current user intent with contextual markov models. In 2013 IEEE 13th International Conference on Data Mining Workshops. IEEE, 391-398.

[20] Chang Liu, Nicholas J Belkin, and Michael J Cole. 2012. Personalization of search results using interaction behaviors in search sessions. In Proceedings of the 35th international ACM SIGIR conference on Research and development in information retrieval. 205-214.

[21] Gary Marchionini. 2006. Exploratory search: from finding to understanding. Commun. ACM 49, 4 (2006), 41-46.

[22] Philipp Mayr. 2016. Sowiport User Search Sessions Data Set (SUSS) (Version 1.0.0). https://doi.org/10.7802/1380

[23] William Noble. 2007. What is a Support Vector Machine? Nature biotechnology 24 (01 2007), 1565-7. https://doi.org/10.1038/nbt1206-1565

[24] J.R. Quinlan. 1987. Simplifying decision trees. International fournal of ManMachine Studies 27, 3 (1987), 221-234. https://doi.org/10.1016/S0020-7373(87) 80053-6

[25] Steffen Rendle, Zeno Gantner, Christoph Freudenthaler, and Lars Schmidt-Thieme 2011. Fast context-aware recommendations with factorization machines. In Proceedings of the 34th international ACM SIGIR conference on Research and development in Information Retrieval. 635-644.

[26] Alice Richardson. 2011. Logistic Regression: A Self-Learning Text, Third Edition by David G. Kleinbaum, Mitchel Klein. International Statistical Review 79 (08 2011), 296-296. https://doi.org/10.2307/41305046
[27] A Shamshad, MA Bawadi, WMA Wan Hussin, TA Majid, and SAM Sanusi. 2005. First and second order Markov chain models for synthetic generation of wind speed time series. Energy 30, 5 (2005), 693-708.

[28] Marc Sloan, Hui Yang, and Jun Wang. 2015. A term-based methodology for query reformulation understanding. Information Retrieval fournal 18, 2 (Mar 2015), 145-165. https://doi.org/10.1007/s10791-015-9251-5

[29] Yang Song and Li-wei He. 2010. Optimal rare query suggestion with implici user feedback. In Proceedings of the 19th international conference on World wide web. 901-910.

[30] Matthijs TJ Spaan. 2012. Partially observable Markov decision processes. In Reinforcement Learning. Springer, 387-414.

[31] Peter D Turney. 2002. The identification of context-sensitive features: A formal definition of context for concept learning. arXiv preprint cs/0212038 (2002)

[32] Armando Vieira. 2016. Predicting online user behaviour using deep learning algorithms. arXiv:1511.06247 [cs.LG]

[33] Ryen W White. 2005. Contextual simulations for information retrieval evaluation. In Proceedings of the ACM SIGIR 2005 Workshop on Information Retrieval in Context (IRiX). 27.

[34] Hui Yang, Dongyi Guan, and Sicong Zhang. 2015. The Query Change Model: Modeling Session Search as a Markov Decision Process. ACM Trans. Inf. Syst. 33, 4, Article 20 (May 2015), 33 pages. https://doi.org/10.1145/2747874 